\title{
Emergency department discharge instructions: a wide variation in practice across Australasia
}

\author{
David McD Taylor, Peter A Cameron
}

\begin{abstract}
Objectives-All patients discharged from the emergency department (ED) should be given discharge instructions (DIs). This study aimed to describe the DI practices of EDs in Australasia and to examine the differences between public and private EDs.

Methods-A voluntary, questionnairebased survey of public and private EDs throughout Australia and New Zealand. Results-58 of 74 (78.4\%) EDs provided instruction notes upon discharge although $51(68.9 \%)$ gave them to only "some" patients. There were no significant differences between the public and private EDs $(p>0.05)$. Thirty seven $(50 \%)$ EDs provided pre-formatted instruction sheets although $22(29.7 \%)$ EDs gave them to only "some" patients. There were no significant differences between the public and private EDs $(p>0.05)$. Only five $(6.8 \%)$ EDs retained a copy of the instruction sheets and no (0\%) ED provided sheets that included all recommended features. Sixty six (89.2\%) EDs provided information sheets although 44 (59.5\%) EDs gave them to only "some" patients. Private EDs gave information sheets to significantly more patients than public EDs ( $\mathrm{p}=0.04)$.
\end{abstract}

Conclusions-The DI practices of individual EDs varied and the rates of DI provision were low. EDs should improve their DI practices. Pre-formatted instruction sheets, containing all recommended features, should be provided to all patients with a copy kept in the medical record. Consideration should be given to the use of DI practices as an ED performance indicator.

(F Accid Emerg Med 2000;17:192-195)

Keywords: emergency; discharge instructions

Royal Melbourne Hospital, Parkville, Victoria, Australia $\mathrm{D}$ McD Taylor

P A Cameron

Correspondence to: Dr McD Taylor, Emergency Department, Royal

Melbourne Hospital,

Parkville, Victoria, Australia 3050 (e-mail:

taylordm@hotmail.com)

Accepted for publication 14 September 1999
All patients discharged to home from the emergency department (ED) should be given instructions for the ongoing management of their illness. It has been suggested that misinterpretation of these instructions can adversely affect compliance, the correct use of medications, treatment, follow up and outcome. ${ }^{1-8}$ There is evidence that improved communication and patient management can be achieved if verbal instructions are complemented by written ED discharge instructions (DIs). ${ }^{2357910}$

Although the nature and format of DIs can vary considerably, three main types have been described and the perceived advantages and disadvantages of each have been discussed. ${ }^{11}$ An instruction note is simply a set of instructions handwritten or typed on plain paper, without the assistance of computer programs. A pre-formatted instruction sheet is a set of instructions handwritten or typed on a pre-formatted document. An example, containing all recommended desirable features, ${ }^{11}$ is shown in figure 1. An information sheet is a pre-printed education and instruction document that describes one specific illness. Only instruction notes and pre-formatted instruction sheets are tailored to the patient.

It has been recommended that all ED patients discharged to home should be given a comprehensive pre-formatted instruction sheet to optimise their post-ED management. ${ }^{7011}$ This may be supplemented by an information sheet if appropriate. ${ }^{11}$ The current DI practices of EDs in Australasia are not known. This study aimed to describe these practices and the features of pre-formatted instruction sheets provided by the EDs. It also aimed to examine the differences in practice between public and private EDs. The study aimed to make recommendations for DI practice based upon the results obtained.

\section{Methods}

The study was a voluntary, questionnairebased survey of public (Australasian College for Emergency Medicine accredited) and private (24 hour/day service and College registered) EDs throughout Australia and New Zealand. This includes all major public EDs and all private EDs. A study questionnaire was mailed to the directors of these EDs in August, 1997. A second mailing was made to those who did not respond initially.

The questionnaire contained two sections. Section one requested demographic data of the participating ED: public or private, site. Section two contained questions that assessed what proportions of patients were provided with instruction notes, pre-formatted instruction sheets and information sheets upon discharge to home. The respondents were required to describe these proportions as either "none", "very few", "some", "about half", "most" or "all patients". Also, respondents were presented with a list of desirable preformatted instruction sheet features. They were required to check which of these features were included in their ED's pre-formatted instruction sheets and to state if their ED retained a copy of the sheets.

The practices of public and private EDs were compared. The Mann-Whitney test 
Royal Hospital Accident \& Emergency Department

Discharge Instruction Sheet

Patient's Name:

This form provides you with initial instructions about your medical care.

Please keep this form and take it with you in case you need further care.

You were seen today by Drs...........................................................(House Officer)

(Specialist)

Your Diagnosis

Expected course of the illness.

Potential complications which may occur...

Instructions:

Medication prescribed (name, dose, frequency, purpose)

$1 .$.

2.

3.

Continue/change your usual medication

Return to the Emergency Department if.

Follow-up with.........................................at Phone No.

Follow-up within..............................days....................weeks.

Instructions given by: Name.

Signature.

I have received and understand these instructions

Name.............

Signature.

Date......

Time.

Figure 1 Example of desirable pre-formatted discharge instruction sheet.

(adjusted for tied responses, $\alpha=0.05, \mathrm{MIN}$ ITAB statistical package) was used to compare groups of EDs where the responses were ordinal. All categorical responses were analysed descriptively.

\section{Results}

The response rate was good with 74 of the 86 EDs $(86.1 \%)$ responding. Fifty seven of the 67 public EDs $(85.1 \%)$ and 17 of the 19 private EDs $(89.5 \%)$ responded. The Australian States and Territories and New Zealand were represented in proportion to their populations (New South Wales 22 respondents, Victoria

Table 1 Proportions of patients discharged home with their own instruction notes

\begin{tabular}{lrllll}
\hline ED type & \multicolumn{1}{l}{ None } & Some & About half & Most & All patients \\
\hline Private $(\mathrm{n}=17)$ & $4(23.5)$ & $12(70.6)$ & $1(5.9)$ & $0(0)$ & $0(0)$ \\
Public $(\mathrm{n}=57)$ & $12(21.1)$ & $39(68.4)$ & $0(0)$ & $4(7.0)$ & $2(3.5)$ \\
Total $(\mathrm{n}=74)$ & $16(21.6)$ & $51(68.9)$ & $1(1.4)$ & $4(5.4)$ & $2(2.7)$
\end{tabular}

Percentages shown in parentheses.

Table 2 Proportions of patients discharged home with their own pre-formatted instruction sheet

\begin{tabular}{lllllll}
\hline ED type & No one & Very few & Some & About half & Most & All patients \\
\hline Private $(\mathrm{n}=17)$ & $10(58.8)$ & $2(11.8)$ & $4(23.5)$ & $1(5.9)$ & $0(0)$ & $0(0)$ \\
Public $(\mathrm{n}=57)$ & $27(47.4)$ & $3(5.2)$ & $18(31.6)$ & $3(5.2)$ & $5(8.8)$ & $1(1.8)$ \\
Total $(\mathrm{n}=74)$ & $37(50.0)$ & $5(6.8)$ & $22(29.7)$ & $4(5.4)$ & $5(6.8)$ & $1(1.3)$ \\
\hline
\end{tabular}

Percentages shown in parentheses.
18, Queensland 14, South Australia 7, Western Australia 4, New Zealand 4, Tasmania 3, Australian Capital Territory 1, Northern Territory 1).

Question 1 asked what proportion of patients were discharged home with their own instruction notes. Table 1 describes the results. Overall, few instruction notes were given out. Most (68.9\%) EDs gave instruction notes to only "some" of their patients and few EDs $(2.7 \%)$ gave them to all patients. There were no significant differences between the public and private EDs ( $p>0.05)$.

Question 2 asked what proportion of patients were discharged home with their own pre-formatted instruction sheet. Table 2 describes the results. Overall, few pre-formatted instruction sheets were given out. Half $(50.0 \%)$ of the EDs gave these sheets to "no-one" and $22(29.7 \%)$ EDs gave them to "some" of their patients. There were no significant differences between the public and private EDs ( $p>0.05)$.

Question 3 asked what proportion of patients were discharged home with their own information sheet. Table 3 describes the results. Overall, more information sheets were given out than instruction notes or preformatted instruction sheets. More than half 
Table 3 Proportions of patients discharged home with their own information sheet

\begin{tabular}{lllllll}
\hline ED type & No one & Very few & Some & About half & Most & All patients \\
\hline Private $^{\star}(\mathrm{n}=17)$ & $0(0)$ & $2(11.7)$ & $8(47.1)$ & $4(23.6)$ & $3(17.6)$ & $0(0)$ \\
Public $^{\star}(\mathrm{n}=57)$ & $8(14.0)$ & $5(8.8)$ & $36(63.2)$ & $2(3.5)$ & $5(8.8)$ & $1(1.7)$ \\
Total $(\mathrm{n}=74)$ & $8(10.8)$ & $7(9.5)$ & $44(59.5)$ & $6(8.1)$ & $8(10.8)$ & $1(1.3)$ \\
\hline
\end{tabular}

* Significant difference between subgroups $(\mathrm{p}<0.05)$. Percentages shown in parentheses.

Table 4 Desirable features included on ED pre-formatted instruction sheets

\begin{tabular}{llll}
\hline Desirable features & $\begin{array}{l}\text { Public ED } \\
(n=26)\end{array}$ & $\begin{array}{l}\text { Private ED } \\
(n=4)\end{array}$ & $\begin{array}{l}\text { Total } \\
(n=30)\end{array}$ \\
\hline Patient's name & $20(76.9)$ & $3(75.0)$ & $23(76.7)$ \\
Advice on the use of the instruction sheet & $13(50.0)$ & $3(75.0)$ & $16(53.3)$ \\
Names(s) of the treating ED doctor(s) & $14(53.9)$ & $4(100)$ & $18(60.0)$ \\
Diagnosis & $18(69.2)$ & $4(100)$ & $22(73.3)$ \\
Expected course of the illness & $14(53.9)$ & $4(100)$ & $18(60.0)$ \\
Potential complications of the illness & $17(65.4)$ & $4(100)$ & $21(70.0)$ \\
Indications for returning to the ED & $21(80.1)$ & $4(100)$ & $25(83.3)$ \\
General treatment instructions & $22(84.6)$ & $3(75.0)$ & $25(83.3)$ \\
New medication instructions & $12(46.2)$ & $1(25.0)$ & $13(43.3)$ \\
Usual medication instructions & $7(26.9)$ & $0(0)$ & $7(23.3)$ \\
Name of person to review illness & $12(46.2)$ & $2(50.0)$ & $14(46.7)$ \\
Timing for review of illness & $12(46.2)$ & $3(75.0)$ & $15(50.0)$ \\
Signature of person providing instruction sheet & $15(57.7)$ & $3(75.0)$ & $18(60.0)$ \\
Statement of understanding of instruction sheet & $1(3.9)$ & $0(0)$ & $1(3.3)$ \\
Signature of person receiving instruction sheet & $2(7.7)$ & $0(0)$ & $2(6.7)$ \\
Date and time & $11(42.3)$ & $1(25.0)$ & $12(40.0)$ \\
\hline Percentages shown in parentheses. & & &
\end{tabular}

Percentages shown in parentheses.

$(59.5 \%)$ of the EDs gave information sheets to "some" patients and only eight (10.8\%) EDs gave the sheets to "no-one". Private EDs gave out information sheets to a significantly greater proportion of patients than public EDs $(\mathrm{p}=0.04)$.

Questions 4 and 5 could be answered only by those EDs that provided pre-formatted instruction sheets. Of the 30 public and seven private EDs that provided these sheets, four public and three private EDs did not respond to these questions. Question 4 presented a list of desirable pre-formatted instruction sheet features ${ }^{11}$ and asked respondents to check which of these were included in their ED's sheet. The results are described in table 4 . Most EDs included the names of the patient and doctor, the illness diagnosis, expected course and complications, indications for return to the $\mathrm{ED}$, general treatment instructions, and the signature of the person providing the instructions. About half of the EDs provided advice on the use of the instruction sheet, new medication instructions, and instructions for patient review. Few EDs included instructions for the use of the patient's usual medications, a statement of understanding, signature of the person receiving the instruction sheet, or the date and time. The differences between public and private EDs were not examined statistically as the number of private EDs responding to this question was small.

Question 5 asked whether the EDs kept a copy of the pre-formatted instruction sheets in the patients' medical record. Five of the 27 $(18.5 \%)$ responding public EDs and zero of the four $(0 \%)$ responding private EDs kept a copy of the instruction sheets.

\section{Discussion}

The study had a good response rate with appropriate representation of EDs across Australia and New Zealand. However, the conclusions may be limited by inaccuracies in the data. It is possible that selection bias impacted upon the results. As only ACEM accredited public and 24 hour ACEM registered private EDs were included in the study, the results may not be representative of all EDs in Australia and New Zealand. Furthermore, the proportions reported by the ED directors were only impressions of their EDs communication practices and unlikely to be based on actual figures. It is probable that many EDs do not maintain a record of GP communications or the mode of these communications. Hence, the reported proportions may be under or over-estimates of actual practices. However, while the proportions reported may not be individually accurate, the overall impression of current DI practices seems clear.

Although the practices of individual EDs varied considerably, the rates at which DIs were provided were low overall and no single type of DI was provided consistently to more than "some" of the patients. Hence, discharge instructions must have been verbal for many patients, a type of instruction that may be inadequate. ${ }^{2} 35791012$ Also, most EDs used at least two types of DIs. Many EDs reported the use of all three types and patients discharged from these EDs could receive any, or a combination, of the three DIs. It is concluded that the rates of provision of DIs were inadequate and that there was no standard DI practice.

Information sheets and instruction notes were the most commonly provided types of DI. The finding that private EDs provided statistically more information sheets than public EDs may represent an effort by the private EDs to optimise patient satisfaction and management, in part, through the use of DIs.

Pre-formatted instruction sheets were provided by only about half of EDs. This practice is not in compliance with the recommendation that a comprehensive, pre-formatted information sheet, supplemented by an information sheet if appropriate, should be provided to all patients. $^{71011}$

The comprehensive, pre-formatted instruction sheet (see Taylor and Cameron ${ }^{11}$ ) has many advantages. Simple language, used in all pre-formatted text to improve readability, can still be comprehensive and inclusive of all desirable features. If the text is completed under specific named headings, patient recall is increased. ${ }^{10^{13} 14}$ The sheets are tailored to the patient, may contain less physician writing but more information than unstructured instruction notes, oblige the writer to be concise, allow for quick interpretation and transfer of information to computerised patient records, and are preferred by GPs. ${ }^{15-20}$ Finally, the structured nature may assist junior staff by prompting them to consider all relevant components of the post-ED management that may otherwise go neglected. ${ }^{1021}$

The evaluation of pre-formatted instruction sheet content shows that recommended desirable features were absent from the sheets of many EDs. It is particularly notable that almost no instruction sheet contained a 
"statement of understanding" of the sheet or provision for the "signature of the person receiving the sheet". Furthermore, very few EDs retained a copy of the instruction sheets provided. It is concluded that, even if preformatted instruction sheets were provided, many did not contain sufficient information for optimum post-ED patient management and the sheet's medico-legal, quality assurance and medical record usefulness were likely to be limited.

It is recommended that EDs improve their discharge instruction practices. Pre-formatted instruction sheets should be provided to all patients discharged to home and a copy should be kept in the patients' medical records. These sheets should include all the desirable features previously described. ${ }^{11}$ Information sheets could be used to provide supplemental information if required. It is recommended that consideration be given to the establishment of state or national guidelines that aim to achieve best practices of DI documentation. The widespread use of standardised DI practices would also permit quality assurance of discharge procedures and DIs could be used as an ED performance indicator.

Contributors

Dr David Taylor initiated and formulated the primary study hypothesis, coordinated the statistical analysis and was primary author. Dr Peter Cameron was involved in study design, collection of data and was second author.

Funding: none:

Conflicts of interest: none.

1 Powers RD. Emergency department patient literacy and the readability of patient-directed materials. Ann Emerg Med 1988;17:124-6

2 Spandorfer JM, Karras DJ, Hughes LA, et al. Comprehension of discharge instructions by patients in an urban emergency department. Ann Emerg Med 1995;25:71-4.

3 Chacon D, Kissoon N, Rich S. Education attainment level of caregivers versus readability level of written instructions in a pediatric emergency department. Pediatr Emerg Care 1994;10:144-9.

4 Gerson LW, Counsell SR, Fontanarosa PB, et al. Case finding for cognitive impairment in elderly emergency department patients. Ann Emerg Med 1994;23:813-17.

5 Mayeaux EJ Jr, Murphy PW, Arnold C, et al. Improving patient education for patients with low literacy skills. Am Fam Physician 1996;53:205-11.

6 Thomas EJ, Burstin HR, O'Neil AC, et al. Patient noncompliance with medical advice after the emergency department visit. Ann Emerg Med 1996;27:49-55.

7 Vukmir RB, Kremen R, Ellis GL, et al. Compliance with emergency department referral: the effect of computerized discharge instructions. Ann Emerg Med 1993;22:819-23.

8 Crane JA. Patient comprehension of doctor-patient communication on discharge from the emergency department. $\mathcal{f}$ Emerg Med 1997;15:1-7.

9 Williams DM, Counselman FL, Caggiano CD. Emergency department discharge instructions and patient literacy: a problem of disparity. Am f Emerg Med 1996;14:19-22.

10 Isaacman DJ, Purvis K, Gyuro J, et al. Standardized instructions: do they improve communication of discharge information from the emergency department? Pediatrics 1992;89 (part 2):1204-8.

11 Taylor DMcD, Cameron PA. Discharge instructions for emergency department patients: what should we provide? 7 Accid Emerg Med 2000; 17:86-90.

12 Grover G, Berkowitz CD, Lewis RJ. Parental recall after a visit to the emergency department. Clin Pediatr 1994;33: 194-201.

13 Bower GH, Clark MC, Lesgold AM, et al. Hierarchical retrieval schemes in recall of categorized word lists. Fournal of Verbal Learning and Verbal Behaviour 1969;8:323-43.

14 Ley P, Bradshaw PW, Eaves D, et al. A method for increasing patients' recall of information presented by doctors. Psychol Med 1973;3:217-20.

15 Montalto M. Letters to go: general practitioners' referral letters to an accident and emergency department. Med $\mathcal{F}$ Aust 1991;155:374-7.

16 Kelly A-M. A study of the content and clarity of general practitioner referral letters to an emergency department. NZ Med F 1993;106:362-3.

17 Wass AR, Illingworth RN. What information do general practitioners want about accident and emergency patients? f Accid Emerg Med 1996;13:406-8

18 Rawal J, Barnett P, Lloyd BW. Use of structured letters to improve communication between hospital doctors and general practitioners. BM7 1993;307:1044.

19 Castleden WM, Stacey MC, Norman PE, et al. General practitioners' attitudes to computer-generated surgical dispractitioners' attitudes to computer-generated
charge letters. Med 7 A A ust 1992;157:380-2.

20 Jenkins S, Arroll B, Hawken S, et al. Referral letters: are Jenkins S, Arroll B, Hawken S, et al. Referral letter
form letters better? Br f Gen Pract 1997;47:107-8.

21 Logan PD, Schwab RA, Salomone JA III, et al. Patient understanding of emergency department discharge instructions. South Med F 1996;89:770-4. 